Pacific

Journal of

Mathematics

ANALYTIC AND TOPOLOGICAL INVARIANTS ASSOCIATED TO NOWHERE ZERO VECTOR FIELDS

WEIPING ZHANG

Volume $187 \quad$ No. 2

February 1999 


\title{
ANALYTIC AND TOPOLOGICAL INVARIANTS ASSOCIATED TO NOWHERE ZERO VECTOR FIELDS
}

\author{
WEIPING ZHANG
}

In memory of Professor Weishu Shi

\begin{abstract}
We construct skew-adjoint operators associated to nowhere zero vector fields on manifolds with vanishing Euler number. The mod 2 indices of these operators provide potentially new invariants for such manifolds. An odd index theorem for corresponding Toeplitz operators is established. This last result may be viewed as an odd dimensional analogue of the GaussBonnet-Chern theorem.
\end{abstract}

\section{Introduction.}

It is well-known that the classical Gauss-Bonnet-Chern theorem $[\mathbf{C}]$ can be interpreted as an index theorem for de Rham-Hodge operators (cf. [BGV] and $[\mathbf{L M}]$ ) and that it is nontrivial only for even dimensional manifolds.

This paper arose from an attempt to search an odd dimensional analogue of this index theorem.

Recall that the Gauss-Bonnet-Chern theorem is closely related to the famous Poincaré-Hopf index formula expressing the Euler number as the sum of indices of singularities of a tangent vector field.

Now for odd dimensional manifolds, or more generally for manifolds with vanishing Euler number, we take the advantage that another result of Hopf asserts that there always exist nowhere zero vector fields (see e.g. Steenrod $[\mathbf{S}])$.

Thus let $M$ be an odd dimensional oriented closed manifold and let $V$ be a nowhere zero vector field on $M$. Let $\gamma$ be the one dimensional oriented vector bundle over $M$ generated by $V$. Then $T M / \gamma$ carries a canonically induced orientation. Let $e(T M / \gamma)$ be the Euler class of $T M / \gamma$.

For any integral element $\omega$ in $H^{1}(M, \mathbf{Q})$, we will take $\langle\omega e(T M / \gamma),[M]\rangle$ as our substitute for the Euler class appeared in the Gauss-Bonnet-Chern theorem. The main result of this paper gives an analytic formula for this number as the index of certain elliptic Toeplitz operators.

In fact, a general odd index theory has already been developed by BaumDouglas $[\mathbf{B D}]$ who pointed out that the associated index can be computed 
from the original Atiyah-Singer index theorem [AS1]. Thus, our result provides a new example for this theory.

Although our index formula can be deduced from the Atiyah-Singer index theorem in its general form, here we will instead develop a purely analytic approach by emphasizing the heat kernel aspects of the index theory. See Section 3 for more details.

Of particular interests is that the operators we construct in Section 2 are in its real form skew-adjoint. Thus, according to Atiyah and Singer [AS2], they would provide interesting mod 2 invariants for manifolds with vanishing Euler number. We will however leave the possible systematic study to elsewhere.

It might also be interesting to note that on the topological side, an odd analogue of the Poincaré-Hopf index theorem has been proved by Geoghegan and Nicas [GN, Theorem 3.1].

This paper is organized as follows. In Section 1, we recall some algebraic preliminaries. In Section 2, we construct the skew-adjoint operators and study its basic properties. In Section 3, we prove an odd index theorem associated to the operators constructed in Section 2. Finally in Section 4 we apply the results of Section 3 to give an analytic interpretation of the above mentioned invariants $\langle\omega e(T M / \gamma),[M]\rangle$.

Acknoledgements. The author would like to thank Mai Zhou for stimulating discussions.

\section{Algebraic preliminaries.}

In this section, we describe the basic algebraic data.

Let $E$ be an oriented Euclidean vector space. If $e \in E$, let $e^{*} \in E^{*}$ corresponds to $e$ by the scalar product of $E$. If $e \in E$, let $c(e), \hat{c}(e)$ be the operators acting on the exterior algebra $\wedge\left(E^{*}\right)$,

$$
\begin{aligned}
& c(e)=e^{*} \wedge-i_{e}, \\
& \hat{c}(e)=e^{*} \wedge+i_{e},
\end{aligned}
$$

where $e^{*} \wedge$ and $i_{e}$ are the standard notation for exterior and inner multiplications. If $e, e^{\prime} \in E$, the following identities hold,

$$
\begin{aligned}
& c(e) c\left(e^{\prime}\right)+c\left(e^{\prime}\right) c(e)=-2\left\langle e, e^{\prime}\right\rangle, \\
& \hat{c}(e) \hat{c}\left(e^{\prime}\right)+\hat{c}\left(e^{\prime}\right) \hat{c}(e)=2\left\langle e, e^{\prime}\right\rangle, \\
& c(e) \hat{c}\left(e^{\prime}\right)+\hat{c}\left(e^{\prime}\right) c(e)=0 .
\end{aligned}
$$

If we view $\wedge\left(E^{*}\right)=\wedge^{\text {even }}\left(E^{*}\right) \oplus \wedge^{\text {odd }}\left(E^{*}\right)$ as a $\mathbf{Z}_{2}$-graded space, then clearly, $c(e), \hat{c}(e)$ are odd elements of $\operatorname{End}\left(\wedge\left(E^{*}\right)\right)$. Also, $\operatorname{End}\left(\wedge\left(E^{*}\right)\right)$ is generated as an algebra by 1 and the $c(e), \hat{c}(e)$ 's.

Let $e_{1}, \ldots, e_{n}$ be an orthonormal base of $E$. 
Proposition 1.1. Among the monomials in the $c\left(e_{i}\right), \hat{c}\left(e_{i}\right)^{\prime} s$, only $c\left(e_{1}\right) \hat{c}\left(e_{1}\right) \ldots c\left(e_{n}\right) \hat{c}\left(e_{n}\right)$ has a nonzero supertrace. Moreover,

$$
\operatorname{Tr}_{s}\left[c\left(e_{1}\right) \hat{c}\left(e_{1}\right) \ldots c\left(e_{n}\right) \hat{c}\left(e_{n}\right)\right]=(-2)^{n} .
$$

For a proof of this Proposition, see [BZ, Section 4d)].

We now assume $n=2 m+1$.

Let $\hat{c}^{\text {odd }}(E)$ be the algebra generated by monomials of the type

$$
c_{I, J}=c\left(e_{i_{1}}\right) \ldots c\left(e_{i_{k}}\right) \hat{c}\left(e_{j_{1}}\right) \ldots \hat{c}\left(e_{j_{l}}\right),
$$

where both $k$ and $l$ are odd integers. Then $\hat{c}^{\text {odd }}(E)$ preserves $\wedge^{\text {even }}\left(E^{*}\right)$ and $\wedge^{\text {odd }}\left(E^{*}\right)$. We will view $\hat{c}^{\text {odd }}(E)$ as a subalgebra of $\operatorname{End}\left(\wedge^{\text {even }}\left(E^{*}\right)\right)$.

Proposition 1.2. Among the monomials of the type (1.4), only $c\left(e_{1}\right) \hat{c}\left(e_{1}\right) \ldots c\left(e_{n}\right) \hat{c}\left(e_{n}\right)$ has a nonzero trace on $\wedge^{\text {even }}\left(E^{*}\right)$. Moreover,

$$
\operatorname{Tr}\left[c\left(e_{1}\right) \hat{c}\left(e_{1}\right) \ldots c\left(e_{n}\right) \hat{c}\left(e_{n}\right)\right]=-2^{2 m} .
$$

Proof. We consider an element of the form (1.4). We assume that no two of $j_{t}$ 's are equal to each other. As $l$ is odd, the set $\left\{j_{1}, \ldots, j_{l}\right\}$ is not empty. We can assume without loss of generality that $j_{1}=1$.

Let $E^{\prime}$ be the subspace of $E$ generated by the $e_{i}$ 's with $i>1$. Then one clearly has

$$
\wedge^{\text {even }}\left(E^{*}\right)=\wedge^{\text {even }}\left(E^{\prime *}\right) \oplus \wedge^{1}\left(\left[e_{1}\right]^{*}\right) \otimes \wedge^{\text {odd }}\left(E^{\prime *}\right) .
$$

Now there are two possibilities.

(i) 1 does not appear in the set $i_{1}, \ldots, i_{k}$. Then one clearly has $\operatorname{Tr}\left[c_{I, J}\right]=0$.

(ii) We can assume without loss of generality that $i_{1}=1$ and that none of $i_{s}, s>1$ is equal to 1 . Then

$$
c_{I, J}=(-1)^{k-1} c\left(e_{1}\right) \hat{c}\left(e_{1}\right) c\left(e_{i_{2}}\right) \ldots c\left(e_{i_{k}}\right) \hat{c}\left(e_{j_{2}}\right) \ldots \hat{c}\left(e_{j_{l}}\right) .
$$

And one verifies directly that

$$
\operatorname{Tr}\left[c_{I, J}\right]=(-1)^{k} \operatorname{Tr}_{s}\left[\left.c\left(e_{i_{2}}\right) \ldots c\left(e_{i_{k}}\right) \hat{c}\left(e_{j_{2}}\right) \ldots \hat{c}\left(e_{j_{l}}\right)\right|_{\wedge\left(E^{\prime *}\right)}\right],
$$

reducing the problem to Proposition 1.1.

\section{Skew-adjoint operators associated to nowhere zero vector fields.}

Let $M$ be a compact oriented manifold. We make the assumption that the Euler number of $M$ vanishes.

By a classical result of Hopf, there exists a nowhere zero vector field on $M$. That is, a vector field $V$ on $M$ such that $V(x) \neq 0$ for all $x \in M$. Without loss of generality we will always assume $V$ is smooth.

If $V_{1}, V_{2}$ are two nowhere zero vector fields on $M$, we say $V_{1}$ is homotopic to $V_{2}$ if there is a smooth family of nowhere zero vector fields $V(t), 0 \leq t \leq 1$ with $V(0)=V_{1}, V(1)=V_{2}$. 
We now fix a nowhere zero vector field $V$ on $M$.

Let $g^{T M}$ be a metric on $M$. We make the assumption that

$$
\|V\|_{g^{T M}}=1 \text {. }
$$

In fact, for any metric $g^{T M}$, we can find a positive function on $M$ so that $f V$ verifies (2.1).

Let $d$ as usual be the exterior derivation acting on $\Gamma\left(\wedge\left(T^{*} M\right)\right)$. Let ${ }^{*}$ be the Hodge star operator of $g^{T M}$.

Let $\langle$,$\rangle be the inner product on \Gamma\left(\wedge\left(T^{*} M\right)\right)$ defined by

$$
\langle\alpha, \beta\rangle=\int_{M} \alpha \wedge * \beta, \quad \alpha, \beta \in \Gamma\left(\wedge\left(T^{*} M\right)\right) .
$$

Let $\delta=d^{*}$ be the formal adjoint of $d$ with respect to the inner product (2.2).

The Clifford actions $c, \hat{c}$ in Section 1 can now be defined for $\wedge\left(T^{*} M\right)$ in the same way. Thus $\hat{c}(V)$ acts on $\wedge\left(T^{*} M\right)$ and interchanges $\wedge^{\text {even }}$ and $\wedge^{\text {odd }}$.

Definition 2.1. The operator $D_{V}$ is the operator acting on $\Gamma\left(\wedge^{\text {even }}\left(T^{*} M\right)\right)$ defined by

$$
D_{V}=\frac{1}{2}(\hat{c}(V)(d+\delta)-(d+\delta) \hat{c}(V)) .
$$

Since the Clifford actions $c$ and $\hat{c}$ anticommute with each other, one verifies easily that $D_{V}$ is a (real) skew-adjoint elliptic first order differential operator. To be more precise, if $e_{1}, \ldots, e_{n}$ is an orthonormal base of $T M$ and $\nabla^{T M}$ is the Levi-Civita connection of $g^{T M}$, then one has the following formula for $D_{V}$.

Proposition 2.2. The following identity holds,

$$
D_{V}=\hat{c}(V)(d+\delta)-\frac{1}{2} \sum_{i} c\left(e_{i}\right) \hat{c}\left(\nabla_{e_{i}}^{T M} V\right)
$$

Proof. Clearly,

$$
d+\delta=\sum_{i} c\left(e_{i}\right) \nabla_{e_{i}}^{\wedge \text { even }\left(T^{*} M\right)}
$$

where $\nabla^{\wedge^{\text {even }}\left(T^{*} M\right)}$ is the Euclidean connection on $\wedge^{\text {even }}\left(T^{*} M\right)$ induced canonically by $\nabla^{T M}$.

From (2.3) and (2.5), one deduces that

$$
\begin{aligned}
D_{V} & =\hat{c}(V)(d+\delta)-\frac{1}{2}(\hat{c}(V)(d+\delta)+(d+\delta) \hat{c}(V)) \\
& =\hat{c}(V)(d+\delta)-\frac{1}{2} \sum_{i} c\left(e_{i}\right) \hat{c}\left(\nabla_{e_{i}}^{T M} V\right) .
\end{aligned}
$$


Now according to Atiyah and Singer [AS2], for any real skew-adjoint elliptic operator $D$, the dimension of the kernel of $D$ is a mod 2 homotopy invariant (cf. also Lawson-Michelsohn $[\mathbf{L M}]$ for an exposition). This is the so called mod 2 index of $D$.

Definition 2.3. Let $\alpha(V)$ be the mod 2 index of $D_{V}$ :

$$
\alpha(V) \equiv \operatorname{dim} \operatorname{ker} D_{V} \quad(\bmod 2) .
$$

Clearly, $\alpha(V)$ depends only on the homotopy class of $V$. The AtiyahSinger mod 2 index theorem [AS2] provides a purely topological formula for $\alpha(V)$.

If we denote by $\mathrm{NZ}(M)$ the set of homotopy classes of nowhere zero vector fields on $M$, then $\alpha$ defines a map

$$
\alpha: \mathrm{NZ}(M) \longrightarrow \mathbf{Z}_{2} \text {. }
$$

Example 2.4. Take $M=S^{1} \times Y$ and assume $M$ has a product metric. Let $V$ be the unit vector field on $S^{1}$. Then $V$ lifts to a unit vector field on $M$ in an obvious way. One verifies easily that $\alpha(V) \equiv \chi(Y)(\bmod 2)$.

From Example 2.4, we see that the map $\alpha$ is nontrivial for manifolds of odd dimensions $4 q+1$. It would be very interesting if this map is also nontrivial in other dimensions. In particular, they might provide new invariants for 3manifolds, as well as for 4-manifolds with vanishing Euler number. Anyway we will leave the possible systematic study to elsewhere.

Here instead, we prove the following result.

Theorem 2.5. If $\operatorname{dim} M=4 q+1$, then the map $\alpha$ in (2.7) is a constant map with value the Kervaire semi-characteristic.

Proof. Recall that the Kervaire semi-characteristic is defined by

$$
k(M)=\sum_{i=0}^{2 q} \operatorname{dim} H^{2 i}(M, \mathbf{R}) \quad(\bmod 2) .
$$

As $\operatorname{dim} M=4 q+1, k(M)$ has the following mod 2 index interpretation (cf. Atiyah-Singer [AS2]).

Let $D_{R}$ be the operator defined by

$$
D_{R}=\hat{c}\left(e_{1}\right) \ldots \hat{c}\left(e_{4 q+1}\right)(d+\delta): \Gamma\left(\wedge^{\text {even }}\left(T^{*} M\right)\right) \longrightarrow \Gamma\left(\wedge^{\text {even }}\left(T^{*} M\right)\right) .
$$

Then one verifies easily that $D_{R}$ is a real skew-adjoint elliptic operator with

$$
\operatorname{dim} \operatorname{ker} D_{R} \equiv k(M) \quad(\bmod 2) \text {. }
$$

Now for any unit vector field $V$ of $\left(T M, g^{T M}\right)$, set

$$
D_{R}^{\prime}=D_{R}-\frac{1}{2} \hat{c}(V) \hat{c}\left(e_{1}\right) \ldots \hat{c}\left(e_{4 q+1}\right) \sum_{i} c\left(e_{i}\right) \hat{c}\left(\nabla_{e_{i}}^{T M} V\right) .
$$


Also one verifies that

$$
\left\langle\nabla^{T M} V, V\right\rangle=0 .
$$

From $(2,12)$, one finds that the elliptic operator $D_{R}^{\prime}$ is also real skewadjoint. Furthermore, one has the following family of real skew-adjoint elliptic operators,

$$
D_{R}(u)=(1-u) D_{R}+u D_{R}^{\prime}, \quad 0 \leq u \leq 1 .
$$

By the homotopy invariance of the $\bmod 2$ indices ([AS2]), and by (2.4), (2.9), (2.11) and (2.12), one then has

$$
\begin{aligned}
\operatorname{dim} \operatorname{ker} D_{R} & \equiv \operatorname{dim} \operatorname{ker} D_{R}^{\prime} \quad(\bmod 2) \\
& =\operatorname{dim} \operatorname{ker} D_{V} .
\end{aligned}
$$

From (2.14), (2.10), one deduces that

$$
\operatorname{dim} \operatorname{ker} D_{V} \equiv k(X) \quad(\bmod 2) .
$$

The proof of Theorem 2.5 is completed.

Remark 2.6. The above argument does not work for dimensions $4 q-1$. Thus it remains an interesting question that whether the map $\alpha$ would still be a constant map for 3-manifolds.

\section{An odd index theorem for nowhere zero vector fields.}

In this Section, we prove the main result of this paper, which is an odd index theorem for the operators $D_{V}$ constructed in Section 2. We assume in this section that $M$ is of odd dimension.

Recall that the odd index theory for self-adjoint elliptic operators has been developed by Baum and Douglas in [BD]. The index theorem we will prove is for the operator $\sqrt{-1} D_{V}$ and can in fact as in [BD] be obtained by an application of the Atiyah-Singer index theorem [AS1] for elliptic pseudodifferential operators. However, here we prefer to give a direct geometric proof of our result. This proof consists of two steps. The first step is to use a theorem of Booss and Wojciechowski $[\mathbf{B W}]$, reducing the problem to a computation of a spectral flow. This spectral flow is then evaluated in the second step by heat equation methods.

This Section is organized as follows. In a), we state the main result of this Section. In b), we reduce the problem to computations of spectral flows. In c), we give an expression of the spectral flow in terms of heat kernels, and in d) we state a result computing the asymptotic expansion constant appearing in the formula in c). In e), we prove some Lichnerowicz type formulas. These formulas will be used in $\mathrm{f}$ ) to give the proof of the result stated in d). 


\section{a). An index theorem for certain Toeplitz operators.}

Recall that $V$ is a unit vector field on an oriented compact odd dimensional Riemannian manifold $M$. We consider the operator $D_{V}=\frac{1}{2}(\hat{c}(V)(d+\delta)-$ $(d+\delta) \hat{c}(V))$ constructed in Section 2 .

In order to apply the ideas of odd index theory for self-adjoint operators, we should complexify our geometric data. So in this Section we consider the exterior algebra bundle with complex coefficients $\wedge_{\mathbf{C}}\left(T^{*} M\right)=\wedge\left(T^{*} M\right) \otimes \mathbf{C}$. Without confusion we still denote it by $\wedge\left(T^{*} M\right)$. The inner product $\langle$, now should be modified accordingly,

$$
\langle\alpha, \beta\rangle=\int_{M} \alpha \wedge \overline{* \beta}, \quad \alpha, \beta \in \wedge\left(T^{*} M\right) .
$$

Definition 3.1. Let $\tilde{D}_{V}$ be the operator $\sqrt{-1} D_{V}$ acting on $\Gamma\left(\wedge^{\text {even }}\left(T^{*} M\right)\right)$.

Clearly, $\tilde{D}_{V}$ is a self-adjoint first order elliptic differential operator. We use the same symbol to denote its closed extension on $L^{2}\left(\wedge^{\text {even }}\left(T^{*} M\right)\right)$.

Let $L_{+}^{2}\left(\wedge^{\text {even }}\left(T^{*} M\right)\right)$ be the direct sum of eigenspaces of $\tilde{D}_{V}$ associated to nonnegative eigenvalues. Denote by $P_{+}$the orthogonal projection operator from $L^{2}\left(\wedge^{\text {even }}\left(T^{*} M\right)\right)$ to $L_{+}^{2}\left(\wedge^{\text {even }}\left(T^{*} M\right)\right)$.

Let $\mathbf{C}^{N}$ be a trivial complex vector bundle over $M$ carrying the trivial metric and connection. Then $\tilde{D}_{V}$ extends trivialy as an operator acting on $\Gamma\left(\wedge^{\text {even }}\left(T^{*} M\right) \otimes \mathbf{C}^{N}\right)$.

Let $g: M \rightarrow U(N)$ be a smooth map from $M$ to the unitary group $U(N)$. Then $g$ extends to an action on $\wedge^{\text {even }}\left(T^{*} M\right) \otimes \mathbf{C}^{N}$ as $\operatorname{Id}_{\wedge \text { even }}\left(T^{*} M\right) \otimes g$. We still note this action by $g$.

Definition 3.2. Let $T_{V, g}$ be the operator

$$
T_{V, g}=\left(P_{+} \otimes \operatorname{Id}_{\mathbf{C}^{N}}\right) g: L_{+}^{2}\left(\wedge^{\text {even }}\left(T^{*} M\right) \otimes \mathbf{C}^{N}\right) \rightarrow L_{+}^{2}\left(\wedge^{\text {even }}\left(T^{*} M\right) \otimes \mathbf{C}^{N}\right) .
$$

This is the so-called Toeplitz operator associated to $\tilde{D}_{V}$ and $g$.

Since $g$ is invertible, one verifies that $T_{V, g}$ is a bounded Fredholm operator between Hilbert spaces.

Let $\gamma$ be the one dimensional oriented vector bundle over $M$ generated by $V$. Let $E$ be the subbundle of $T M$ orthogonal to $\gamma$. Then $E$ carries a canonically induced orientation so that $o(\gamma, E)=o(T M)$. Take a metric on $E$ and let $\nabla$ be a Euclidean connection for this metric on $E$. Let $R$ be the curvature of $\nabla$.

The main result of this Section can be stated as follows.

Theorem 3.3. The following identity holds,

$$
\operatorname{ind}\left(T_{V, g}\right)=\frac{1}{2 \pi \sqrt{-1}} \int_{M} \operatorname{Tr}\left[g^{-1} d g\right] \operatorname{Pf}\left(\frac{R}{2 \pi}\right) .
$$


Theorem 3.3 can be proved by an application of the Atiyah-Singer index theorem [AS1] as in Baum-Douglas [BD]. In the rest of this Section, we will give a geometric proof of (3.3).

\section{b). Toeplitz operators and spectral flow.}

Note that by now $\tilde{D}_{V}$ is a self-adjoint elliptic operator acting on $\Gamma\left(\wedge^{\text {even }}\left(T^{*} M\right) \otimes \mathbf{C}^{N}\right)$.

Since $g$ is unitary, the operator

$$
\tilde{D}_{V, g}=g^{-1} \tilde{D}_{V} g
$$

is also a self-adjoint operator on $\Gamma\left(\wedge^{\text {even }}\left(T^{*} M\right) \otimes \mathbf{C}^{N}\right)$.

Let $\tilde{D}_{V}(u)$ be the family of self-adjoint operators

$$
\tilde{D}_{V}(u)=(1-u) \tilde{D}_{V}+u \tilde{D}_{V, g}, \quad 0 \leq u \leq 1 .
$$

Let $\operatorname{sf}\left\{\tilde{D}_{V}(u), 0 \leq u \leq 1\right\}$ be the spectral flow of the family $\left\{\tilde{D}_{V}(u)\right\}_{0 \leq u \leq 1}$ in the sense of Atiyah, Patodi and Singer [APS2].

Then one has:

Theorem 3.4. The following identity holds,

$$
\operatorname{ind}\left(T_{V, g}\right)=-\operatorname{sf}\left\{\tilde{D}_{V}(u), 0 \leq u \leq 1\right\} .
$$

Proof. Formula (3.6) is a corollary of a general result of Booss and Wojciechowski (cf. [BW, Theorem 17.17]).

\section{c). $\eta$ invariants and spectral flow}

For a self-adjoint first order elliptic differential operator $D$, we will adopt the standard notation $\eta(D)$ for the $\eta$ invariant of $D$ in the sense of Atiyah, Patodi and Singer [APS1]. Let $\bar{\eta}(D)$ be the reduced $\eta$ invariant of $D$, also defined in [APS1]:

$$
\bar{\eta}(D)=\frac{1}{2}(\operatorname{dim} \operatorname{ker}(D)+\eta(D)) .
$$

Let $\tilde{D}_{V}(u), 0 \leq u \leq 1$, be our family of first order elliptic self-adjoint operators. Clearly, for any $u \in[0,1]$,

$$
\frac{\partial}{\partial u} \tilde{D}_{V}(u)=\tilde{D}_{V, g}-\tilde{D}_{V}
$$

is a bounded operator.

By the standard results for heat kernel asymptotics, one has the following asymptotic expansion as $t \rightarrow 0^{+}$,

$$
\operatorname{Tr}\left[\frac{\partial}{\partial u} \tilde{D}_{V}(u) \exp \left(-t \tilde{D}_{V}(u)^{2}\right)\right]=\frac{c_{-\frac{n}{2}}}{t^{\frac{n}{2}}}+\cdots+\frac{c_{-\frac{1}{2}}}{t^{\frac{1}{2}}}+O\left(t^{\frac{1}{2}}\right),
$$

where $n=\operatorname{dim} M$ is the dimension of $M$ and $c_{-\frac{n}{2}}, \ldots, c_{-\frac{1}{2}}$ are smooth functions of $u \in[0,1]$. 
The main result of this subsection can be stated as follows.

Proposition 3.5. The following identity holds,

$$
\operatorname{sf}\left\{\tilde{D}_{V}(u), 0 \leq u \leq 1\right\}=\int_{0}^{1} \frac{c_{-\frac{1}{2}}}{\sqrt{\pi}} d u .
$$

In fact, we will prove the following more general result.

Proposition 3.6. For any $s \in[0,1]$, one has

$$
\operatorname{sf}\left\{\tilde{D}_{V}(u), 0 \leq u \leq s\right\}=\int_{0}^{s} \frac{c_{-\frac{1}{2}}}{\sqrt{\pi}} d u+\bar{\eta}\left(\tilde{D}_{V}(s)\right)-\bar{\eta}\left(\tilde{D}_{V}\right) .
$$

Proof. First of all, (3.11) holds for $s=0$. Let $I$ be the subset of $[0,1]$ such that for any $s \in I,(3.11)$ holds. Then $I$ is not empty.

Take $u_{0} \in I$. Let $\varepsilon_{0}>0$ be sufficiently small so that

$$
\operatorname{Spec}\left(\tilde{D}_{V}\left(u_{0}\right)\right) \bigcap\left[-\varepsilon_{0}, \varepsilon_{0}\right]=\{0\} .
$$

Since the eigenvalues of $\tilde{D}_{V}(u)$ are continuous functions of $u$, there is a sufficiently small neighborhood $U$ of $u_{0}$ in $[0,1]$ such that for any $u \in U, \pm \varepsilon_{0}$ are not eigenvalues of $\tilde{D}_{V}(u)$.

Take any $u \in U$. Let $E_{+}^{u}\left(\epsilon_{0}\right)$ (resp. $E_{-}^{u}\left(-\varepsilon_{0}\right)$ ) be the direct sum of eigenspaces of $\tilde{D}_{V}(u)$ associated to eigenvalues greater than $\varepsilon_{0}$ (resp. less than $\left.-\varepsilon_{0}\right)$. Let $P_{+}^{u}\left(\right.$ resp. $\left.P_{-}^{u}\right)$ be the orthogonal projection operator from $L^{2}\left(\wedge^{\text {even }}\left(T^{*} M\right) \otimes \mathbf{C}^{N}\right)$ onto $E_{+}^{u}\left(\varepsilon_{0}\right)\left(\right.$ resp. $\left.E_{-}^{u}\left(-\varepsilon_{0}\right)\right)$.

Set $P=P^{u}=P_{+}^{u}+P_{-}^{u}$.

Let $s \in \mathbf{C}$ be such that $\operatorname{Re}(s) \gg 0$. Set

$$
\eta_{\varepsilon_{0}}\left(\tilde{D}_{V}(u), s\right)=\frac{1}{\Gamma\left(\frac{s+1}{2}\right)} \int_{0}^{\infty} t^{\frac{s-1}{2}} \operatorname{Tr}\left[P \tilde{D}_{V}(u) \exp \left(-t \tilde{D}_{V}(u)^{2}\right) P\right] d t .
$$

This function extends to a meromorphic function on $\mathbf{C}$, which is holomorphic at $s=0([$ APS1, APS2 $])$.

Since $P^{2}=P$, one has

$$
\left(\frac{\partial}{\partial u} P\right) P+P \frac{\partial}{\partial u} P=\frac{\partial}{\partial u} P .
$$

From (3.14), one gets

$$
P\left(\frac{\partial}{\partial u} P\right) P=0 .
$$

From (3.13)-(3.15) and by proceeding as in Bismut-Freed [BF, (2.31)], one finds that

$$
\Gamma\left(\frac{s+1}{2}\right) \frac{\partial}{\partial u} \eta_{\varepsilon_{0}}\left(\tilde{D}_{V}(u), s\right)
$$




$$
=-s \int_{0}^{\infty} t^{\frac{s-1}{2}}\left\{\operatorname{Tr}\left[P \frac{\partial}{\partial u} \tilde{D}_{V}(u) \exp \left(-t \tilde{D}_{V}(u)^{2}\right) P\right]\right\} d t
$$

On the other hand, since $\frac{\partial}{\partial u} \tilde{D}_{V}(u)$ is a bounded operator, from (3.9) one verifies easily that as $t \rightarrow 0^{+}$, one has the following asymptotic expansion,

$$
\operatorname{Tr}\left[P \frac{\partial}{\partial u} \tilde{D}_{V}(u) \exp \left(-t \tilde{D}_{V}(u)^{2}\right) P\right]=\frac{c_{-\frac{n}{2}}}{t^{\frac{n}{2}}}+\cdots+\frac{c_{-\frac{1}{2}}}{t^{\frac{1}{2}}}+O(1) .
$$

From (3.16), (3.17), one gets

$$
\frac{\partial}{\partial u} \eta_{\varepsilon_{0}}\left(\tilde{D}_{V}(u), 0\right)=-\frac{2 c_{-\frac{1}{2}}}{\sqrt{\pi}} .
$$

Now let $k_{u}$ be the number of negative eigenvalues of $\tilde{D}_{V}(u)$ in $\left(-\varepsilon_{0}, 0\right)$. Then by the definition of spectral flow, one has

$$
\operatorname{sf}\left\{\tilde{D}_{V}(\alpha): \alpha \text { from } u_{0} \text { to } u\right\}=-k_{u} .
$$

Also, by (3.18) one has,

$$
\begin{aligned}
& \bar{\eta}\left(\tilde{D}_{V}(u)\right)-\bar{\eta}\left(\tilde{D}_{V}\left(u_{0}\right)\right) \\
& =\frac{1}{2}\left\{\eta_{\varepsilon_{0}}\left(\tilde{D}_{V}(u), 0\right)-\eta_{\varepsilon_{0}}\left(\tilde{D}_{V}\left(u_{0}\right), 0\right)\right\}-k_{u} \\
& =-\int_{u_{0}}^{u} \frac{c_{-\frac{1}{2}}}{\sqrt{\pi}} d s-k_{u} .
\end{aligned}
$$

Since (3.11) holds for $u_{0}$, by (3.19), (3.20) and by the additivity of spectral flow, it also holds for $u \in U$.

Thus the set $I$ is open in $[0,1]$. A similar argument shows that $I$ is also closed in $[0,1]$. Thus $I=[0,1]$.

Proposition 3.6 is then proved.

Proof of Proposition 3.5. Since $g^{-1} \tilde{D}_{V} g$ is conjugate to $\tilde{D}_{V}$, they have the same spectrum. Thus, one has

$$
\bar{\eta}\left(g^{-1} \tilde{D}_{V} g\right)=\bar{\eta}\left(\tilde{D}_{V}\right)
$$

Proposition 3.5 follows from (3.21) by setting $s=1$ in (3.11).

Remark 3.7. Proposition 3.6 may be seen as a refinement of [BF, Proposition 2.8] in our situation. This kind of refinements is in fact well-known. We include a proof here just for the sake of completeness.

Remark 3.8. By Theorem 3.4 and Proposition 3.5, in order to prove Theorem 3.3 , we need only to evaluate $c_{-\frac{1}{2}}$ for each $u \in[0,1]$. This is the subject of the following subsections. 
d). An asymptotic result involving the heat kernel of $\tilde{D}_{V}(u)^{2}$.

Recall that $E$ is the oriented subbundle of $T M$ such that $E$ is orthogonal to the oriented line bundle $\gamma$ generated by $V$ and that $(\gamma, E)$ has the same orientation as $T M$.

Let

$$
\begin{gathered}
T M=\gamma \oplus E, \\
g^{T M}=g^{\gamma}+g^{E}
\end{gathered}
$$

be the corresponding orthogonal decomposition of the metric $g^{T M}$. Let $R^{T M}$ be the curvature of the Levi-Civita connection $\nabla^{T M}$. Let $P^{E}\left(\right.$ resp. $\left.P^{\gamma}\right)$ be the orthogonal projection from $T M$ to $E$ (resp. $\gamma$ ). Then

$$
\begin{gathered}
\nabla^{\gamma}=P^{\gamma} \nabla^{T M} P^{\gamma}, \\
\nabla^{E}=P^{E} \nabla^{T M} P^{E}
\end{gathered}
$$

are Euclidean connections of $g^{\gamma}, g^{E}$ respectively.

Let $R^{E}=\left(\nabla^{E}\right)^{2}$ be the curvature of $\nabla^{E}$.

For any $u \in[0,1]$, let $Q_{t}^{u}(x, y)$ be the kernel of $\frac{\partial}{\partial u} \tilde{D}_{V}(u) \exp \left(-t \tilde{D}_{V}(u)^{2}\right)$. Let $d v_{M}$ be the volume element on $T M$ with respect to $g^{T M}$.

The main result of this subsection can be stated as follows.

Theorem 3.9. For any $u \in[0,1], x \in M$, the following identity holds,

$$
\lim _{t \rightarrow 0} \sqrt{t} \operatorname{Tr}\left[Q_{t}^{u}(x, x)\right] d v_{M}(x)=\frac{\sqrt{-1}}{2 \sqrt{\pi}} \operatorname{Tr}\left[g^{-1} d g\right] \operatorname{Pf}\left(\frac{R^{E}}{2 \pi}\right) .
$$

By Remark 3.8, Theorem 3.3 is a consequence of Theorem 3.9 and the Chern-Weil theorem (cf. [BGV]) for Euler forms. The rest of this section is devoted to the proof of (3.24).

e). A Lichnerowicz formula for $\tilde{D}_{V}(u)^{2}$.

We write $n=2 m+1$.

Let $e_{0}, \ldots, e_{2 m}$ be an orthonormal base of $T M$.

Proposition 3.10. The following identity holds,

$$
\begin{aligned}
\tilde{D}_{V}(u)^{2}= & \tilde{D}_{V}^{2}+u\left((d+\delta) c\left(g^{-1} d g\right)+c\left(g^{-1} d g\right)(d+\delta)\right) \\
& +\frac{u}{2} \sum_{0}^{2 m} c\left(e_{i}\right) c\left(g^{-1} d g\right) \hat{c}(V) \hat{c}\left(\nabla_{e_{i}}^{T M} V\right) \\
& +\frac{u}{2} \sum_{0}^{2 m} c\left(g^{-1} d g\right) c\left(e_{i}\right) \hat{c}(V) \hat{c}\left(\nabla_{e_{i}}^{T M} V\right) \\
& +u^{2}\left(c\left(g^{-1} d g\right)\right)^{2} .
\end{aligned}
$$


Proof. By (2.4), Definition 3.1, one has

$$
\tilde{D}_{V}=\sqrt{-1} \hat{c}(V)(d+\delta)+\frac{1}{2 \sqrt{-1}} \sum_{0}^{2 m} c\left(e_{i}\right) \hat{c}\left(\nabla_{e_{i}}^{T M} V\right) .
$$

Also,

$$
\begin{aligned}
g^{-1} \tilde{D}_{V} g & =\tilde{D}_{V}+g^{-1}\left[\tilde{D}_{V}, g\right] \\
& =\tilde{D}_{V}+\sqrt{-1} \hat{c}(V) c\left(g^{-1} d g\right) .
\end{aligned}
$$

From (3.5) and (3.27), one has

$$
\tilde{D}_{V}(u)=\tilde{D}_{V}+u \sqrt{-1} \hat{c}(V) c\left(g^{-1} d g\right) .
$$

Thus,

$$
\begin{aligned}
\tilde{D}_{V}(u)^{2}= & \tilde{D}_{V}^{2}+u \sqrt{-1} \tilde{D}_{V} \hat{c}(V) c\left(g^{-1} d g\right) \\
& +u \sqrt{-1} \hat{c}(V) c\left(g^{-1} d g\right) \tilde{D}_{V} \\
& -u^{2} \hat{c}(V) c\left(g^{-1} d g\right) \hat{c}(V) c\left(g^{-1} d g\right) .
\end{aligned}
$$

(3.25) follows from (3.29), (3.26) and (1.2).

Let $\Delta$ be the standard notation for the Bochner Laplacian

$$
\Delta=\sum_{0}^{2 m}\left(\nabla_{e_{i}}^{\wedge^{\text {even }}\left(T^{*} M\right), 2}-\nabla_{\nabla_{e_{i}}^{T M} e_{i}}^{\wedge^{\text {even }}\left(T^{*} M\right)}\right) .
$$

Let $K$ be the scalar curvature of $g^{T M}$.

We also make the assumption that $e_{0}=V$. Then $e_{1}, \ldots, e_{2 m}$ is an orthonormal base of $E$.

Theorem 3.11. The following identity holds,

$$
\text { (3.31) } \begin{aligned}
\tilde{D}_{V}^{2}= & -\Delta+\frac{K}{4} \\
& +\frac{1}{8} \sum_{0 \leq i, j \leq 2 m} \sum_{1 \leq k, l \leq 2 m}\left\langle R^{E}\left(e_{i}, e_{j}\right) e_{l}, e_{k}\right\rangle c\left(e_{i}\right) c\left(e_{j}\right) \hat{c}\left(e_{k}\right) \hat{c}\left(e_{l}\right) \\
& -\frac{1}{2} \hat{c}(V) \hat{c}(\Delta V)-\sum_{0}^{2 m} \hat{c}(V) \hat{c}\left(\nabla_{e_{i}}^{T M} V\right) \nabla_{e_{i}}^{T M}-\frac{1}{4} \sum_{0}^{2 m}\left\|S\left(e_{i}\right) V\right\|^{2} .
\end{aligned}
$$

Proof. By (3.26), one has

$$
\tilde{D}_{V}^{2}=-(\hat{c}(V)(d+\delta))^{2}+\frac{1}{2}\left(\hat{c}(V)(d+\delta) \sum_{0}^{2 m} c\left(e_{i}\right) \hat{c}\left(\nabla_{e_{i}}^{T M} V\right)\right.
$$




$$
\left.+\sum_{0}^{2 m} c\left(e_{i}\right) \hat{c}\left(\nabla_{e_{i}}^{T M} V\right) \hat{c}(V)(d+\delta)\right)-\frac{1}{4}\left(\sum_{0}^{2 m} c\left(e_{i}\right) \hat{c}\left(\nabla_{e_{i}}^{T M} V\right)\right)^{2} .
$$

Now

$$
\begin{aligned}
& (\hat{c}(V)(d+\delta))^{2} \\
& =-(d+\delta)^{2}+\hat{c}(V)((d+\delta) \hat{c}(V)+\hat{c}(V)(d+\delta))(d+\delta) \\
& =-(d+\delta)^{2}+\hat{c}(V) \sum_{0}^{2 m} c\left(e_{i}\right) \hat{c}\left(\nabla_{e_{i}}^{T M} V\right)(d+\delta) .
\end{aligned}
$$

By (3.32), (3.33), one deduces

$$
\begin{aligned}
\tilde{D}_{V}^{2}= & (d+\delta)^{2}+\frac{1}{2}\left\{\hat{c}(V)(d+\delta) \sum_{0}^{2 m} c\left(e_{i}\right) \hat{c}\left(\nabla_{e_{i}}^{T M} V\right)\right. \\
& \left.-\hat{c}(V) \sum_{0}^{2 m} c\left(e_{i}\right) \hat{c}\left(\nabla_{e_{i}}^{T M} V\right)(d+\delta)\right\} \\
& -\frac{1}{4}\left(\sum_{0}^{2 m} c\left(e_{i}\right) \hat{c}\left(\nabla_{e_{i}}^{T M} V\right)\right)^{2} .
\end{aligned}
$$

The middle term in the right hand side of (3.34) can be evaluated as follows,

$$
\begin{aligned}
\hat{c}(V)(d+\delta) \sum_{0}^{2 m} c\left(e_{i}\right) \hat{c}\left(\nabla_{e_{i}}^{T M} V\right)-\hat{c}(V) \sum_{0}^{2 m} c\left(e_{i}\right) \hat{c}\left(\nabla_{e_{i}}^{T M} V\right)(d+\delta) \\
=\hat{c}(V) \sum_{0}^{2 m}\left\{(d+\delta) c\left(e_{i}\right)+c\left(e_{i}\right)(d+\delta)\right\} \hat{c}\left(\nabla_{e_{i}}^{T M} V\right) \\
\quad-\hat{c}(V) \sum_{0}^{2 m} c\left(e_{i}\right)\left\{\hat{c}\left(\nabla_{e_{i}}^{T M} V\right)(d+\delta)+(d+\delta) \hat{c}\left(\nabla_{e_{i}}^{T M} V\right)\right\} \\
=\hat{c}(V) \sum_{0}^{2 m}\left(-2 \nabla_{e_{i}}^{T M}\right) \hat{c}\left(\nabla_{e_{i}}^{T M} V\right)+\hat{c}(V) \sum_{i, j} c\left(e_{j}\right) c\left(\nabla_{e_{j}}^{T M} e_{i}\right) \hat{c}\left(\nabla_{e_{i}}^{T M} V\right) \\
\quad-\hat{c}(V) \sum_{i, j} c\left(e_{i}\right) c\left(e_{j}\right) \hat{c}\left(\nabla_{e_{j}}^{T M} \nabla_{e_{i}}^{T M} V\right) \\
=\hat{c}(V)\left\{-2 \sum_{i} \hat{c}\left(\nabla_{e_{i}}^{T M} V\right) \nabla_{e_{i}}^{T M}-\hat{c}(\Delta V)\right\} \\
\quad+\frac{1}{2} \sum_{i, j} c\left(e_{i}\right) c\left(e_{j}\right) \hat{c}(V) \hat{c}\left(R^{T M}\left(e_{i}, e_{j}\right) V\right) .
\end{aligned}
$$


Let $S$ be defined by

$$
S=\nabla^{T M}-\nabla^{E}-\nabla^{\gamma} .
$$

Then $S$ is a one form taking values in skew-symmetric endomorphisms interchanging $\gamma$ and $E$.

Recall that $e_{0}=V$. We have

$$
\begin{aligned}
& -\left(\sum_{0}^{2 m} c\left(e_{i}\right) \hat{c}\left(\nabla_{e_{i}}^{T M} V\right)\right)^{2} \\
= & \sum_{i, j} c\left(e_{i}\right) c\left(e_{j}\right) \hat{c}\left(\nabla_{e_{i}}^{T M} V\right) \hat{c}\left(\nabla_{e_{j}}^{T M} V\right) \\
= & \sum_{i, j} c\left(e_{i}\right) c\left(e_{j}\right) \hat{c}\left(S\left(e_{i}\right) V\right) \hat{c}\left(S\left(e_{j}\right) V\right) \\
= & \frac{1}{2} \sum_{i, j, k, l}\left(\left\langle S\left(e_{i}\right) V, e_{k}\right\rangle\left\langle S\left(e_{j}\right) V, e_{l}\right\rangle\right. \\
& \left.-\left\langle S\left(e_{j}\right) V, e_{k}\right\rangle\left\langle S\left(e_{i}\right) V, e_{l}\right\rangle\right) c\left(e_{i}\right) c\left(e_{j}\right) \hat{c}\left(e_{k}\right) \hat{c}\left(e_{l}\right) \\
& -\sum_{0}^{2 m}\left\|S\left(e_{i}\right) V\right\|^{2} .
\end{aligned}
$$

Also, from (3.36), one deduces that for $1 \leq k, l \leq 2 m$,

$$
\begin{aligned}
& \left\langle R^{T M}\left(e_{i}, e_{j}\right) e_{l}, e_{k}\right\rangle \\
& =\left\langle R^{E}\left(e_{i}, e_{j}\right) e_{l}, e_{k}\right\rangle+\left\langle S\left(e_{i}\right) S\left(e_{j}\right) e_{l}-S\left(e_{j}\right) S\left(e_{i}\right) e_{l}, e_{k}\right\rangle \\
& =\left\langle R^{E}\left(e_{i}, e_{j}\right) e_{l}, e_{k}\right\rangle+\left\langle S\left(e_{i}\right) e_{l}, V\right\rangle\left\langle S\left(e_{j}\right) e_{k}, V\right\rangle-\left\langle S\left(e_{j}\right) e_{l}, V\right\rangle\left\langle S\left(e_{i}\right) e_{k}, V\right\rangle .
\end{aligned}
$$

On the other hand, by using an obvious extension of the Lichnerowicz formula $[\mathbf{L}]$ for $(d+\delta)^{2}$ (cf. [BZ, Section 4e)] and [BGV]), one has

$$
(d+\delta)^{2}=-\Delta+\frac{K}{4}+\frac{1}{8} \sum_{i, j, k, l}\left\langle R^{T M}\left(e_{i}, e_{j}\right) e_{l}, e_{k}\right\rangle c\left(e_{i}\right) c\left(e_{j}\right) \hat{c}\left(e_{k}\right) \hat{c}\left(e_{l}\right)
$$

(3.31) follows from (3.34), (3.35) and (3.37)-(3.39).

Proposition 3.10 and Theorem 3.11 together gives the following crucial Lichnerowicz type formula.

Theorem 3.12. For any $u \in[0,1]$, the following identity holds,

$$
\begin{aligned}
\tilde{D}_{V}(u)^{2}= & -\Delta+\frac{K}{4} \\
& +\frac{1}{8} \sum_{0 \leq i, j \leq 2 m} \sum_{1 \leq k, l \leq 2 m}\left\langle R^{E}\left(e_{i}, e_{j}\right) e_{l}, e_{k}\right\rangle c\left(e_{i}\right) c\left(e_{j}\right) \hat{c}\left(e_{k}\right) \hat{c}\left(e_{l}\right)
\end{aligned}
$$




$$
\begin{aligned}
& -\frac{1}{2} \hat{c}(V) \hat{c}(\Delta V)-\sum_{0}^{2 m} \hat{c}(V) \hat{c}\left(\nabla_{e_{i}}^{T M} V\right) \nabla_{e_{i}}^{T M} \\
& +u\left((d+\delta) c\left(g^{-1} d g\right)+c\left(g^{-1} d g\right)(d+\delta)\right) \\
& +\frac{u}{2} \sum_{0}^{2 m} c\left(e_{i}\right) c\left(g^{-1} d g\right) \hat{c}(V) \hat{c}\left(\nabla_{e_{i}}^{T M} V\right) \\
& +\frac{u}{2} \sum_{0}^{2 m} c\left(g^{-1} d g\right) c\left(e_{i}\right) \hat{c}(V) \hat{c}\left(\nabla_{e_{i}}^{T M} V\right) \\
& +u^{2}\left(c\left(g^{-1} d g\right)\right)^{2}-\frac{1}{4} \sum_{0}^{2 m}\left\|S\left(e_{i}\right) V\right\|^{2} .
\end{aligned}
$$

\section{f). A proof of Theorem 3.9.}

We will use the method of Bismut and Zhang [BZ, Section 4] to prove Theorem 3.9. In particular, we adopt the notation used there. For example, for any $e \in T M$ and $e^{*} \in T^{*} M$ its corresponding element via $g^{T M}$, we will write as in $[\mathbf{B Z}$, Section 4$]$ that

$$
\hat{c}(e)=\hat{e}^{*} \wedge+i_{\hat{e}} .
$$

For any $t>0, x \in M, e \in T M$ and $e^{*} \in T^{*} M$ its correspondent, set

$$
\begin{aligned}
& c_{t}(e)=\frac{e^{*}}{t^{\frac{1}{4}}} \wedge-t^{\frac{1}{4}} i_{e}, \\
& \hat{c}_{t}(e)=\frac{\hat{e}^{*}}{t^{\frac{1}{4}}} \wedge+t^{\frac{1}{4}} i_{\hat{e}} .
\end{aligned}
$$

Then for any $e, e^{\prime} \in T M$, one has

$$
\begin{aligned}
& c_{t}(e) c_{t}\left(e^{\prime}\right)+c_{t}\left(e^{\prime}\right) c_{t}(e)=-2\left\langle e, e^{\prime}\right\rangle, \\
& \hat{c}_{t}(e) \hat{c}_{t}\left(e^{\prime}\right)+\hat{c}_{t}\left(e^{\prime}\right) \hat{c}_{t}(e)=2\left\langle e, e^{\prime}\right\rangle, \\
& c_{t}(e) \hat{c}_{t}\left(e^{\prime}\right)+\hat{c}_{t}\left(e^{\prime}\right) c_{t}(e)=0 .
\end{aligned}
$$

Furthermore, for any $\alpha \in \operatorname{End}\left(\wedge^{\text {even }}\left(T^{*} M\right)\right)$, if we denote by $\alpha_{t}$ the element obtained from $\alpha$ by replacing $c(e)$ by $c_{t}(e)$ and $\hat{c}(e)$ by $\hat{c}_{t}(e)$, then we have:

Proposition 3.13. If $\alpha \in \operatorname{End}\left(\wedge^{\operatorname{even}}\left(T^{*} M\right)\right)$, then for any $t>0$,

$$
\operatorname{Tr}[\alpha]=(-1)^{m+1} 2^{2 m} t^{m+\frac{1}{2}}\left\{\alpha_{t}\right\}^{\max },
$$

where $\left\{\alpha_{t}\right\}^{\max }$ denotes the coefficient of the monomial $e^{0} \wedge \ldots \wedge e^{2 m} \wedge \hat{e}^{0} \ldots \wedge \hat{e}^{2 m}$ in the expansion of $\alpha_{t}$.

Proof. Equation (3.44) follows from (1.5). 
Now we proceed as in Bismut-Zhang [BZ, Section 4] and Getzler [G].

Let $a>0$ be the injectivity radius of $\left(M, g^{T M}\right)$. Take $\varepsilon$ such that $0<$ $\varepsilon \leq a / 2$. Take $x \in M$. Let $e_{0}, \ldots, e_{2 m}$ be an orthonormal base of $T_{x} M$. We make the assumption that $e_{0}=V(x)$. We identify the open ball $B^{T_{x} M}(0, \varepsilon)$ in $T_{x} M$ with the open ball $B^{M}(x, \varepsilon)$ in $M$ using geodesic coordinates. Then $y \in T_{x} M,|y| \leq \varepsilon$ represents an element of $B^{M}(x, \varepsilon)$. For $y \in T_{x} M,|y| \leq \varepsilon$, we identify $T_{y} M, \mathbf{C}_{y}^{N}$ to $T_{x} M, \mathbf{C}_{x}^{N}$ by parallel transports along the geodesic $t \in[0,1] \rightarrow t y$ with respect to $\nabla^{T M}$ and the trivial connection on $\mathbf{C}^{N}$.

Let $\Gamma^{T M, x}$ be the connection form for $\nabla^{T M}$ in the considered trivialization of TM. By [ABP, Proposition 4.7], we know that

$$
\Gamma_{y}^{T M, x}=\frac{1}{2} R_{x}^{T M}(y, \cdot)+O\left(|y|^{2}\right) .
$$

The induced connection form $\Gamma_{y}^{\wedge^{\text {even }}}\left(T_{x}^{*} M\right)$ on $\wedge^{\text {even }}\left(T_{x}^{*} M\right)$ is given by (3.46)

$$
\Gamma_{y}^{\wedge^{\text {even }}\left(T^{*} M\right), x}=\frac{1}{8} \sum_{i, j}\left(\left\langle R_{x}^{T M}(y, \cdot) e_{i}, e_{j}\right\rangle+O\left(|y|^{2}\right)\right)\left(c\left(e_{i}\right) c\left(e_{j}\right)-\hat{c}\left(e_{i}\right) \hat{c}\left(e_{j}\right)\right) .
$$

The operator $\tilde{D}_{V}(u)^{2}$ now acts on smooth sections of $\left(\wedge^{\text {even }}\left(T^{*} M\right) \otimes \mathbf{C}^{N}\right)_{x}$ over $B^{T_{x} M}(0, \varepsilon)$.

If $h$ is a smooth section of $\left(\wedge^{\text {even }}\left(T^{*} M\right) \otimes \mathbf{C}^{N}\right)_{x}$ over $T_{x} M$, set

$$
T_{t} h(y)=h\left(\frac{y}{\sqrt{t}}\right) \text {. }
$$

Let $K_{t}(u)$ be the operator

$$
K_{t}(u)=T_{t}^{-1} t \tilde{D}(u)^{2} T_{t}
$$

Then $K_{t}(u)$ is a differential operator with coefficients in the algebra spanned by the elements of $\operatorname{End}\left(\wedge^{\text {even }}\left(T_{x}^{*} M\right)\right)$ and $\operatorname{End}\left(\mathbf{C}^{N}\right)_{x}$.

Let $L_{t}(u)$ be the operator obtained from $K_{t}(u)$ by replacing the Clifford variables $c\left(e_{i}\right), \hat{c}\left(e_{i}\right)$ by $c_{t}\left(e_{i}\right), \hat{c}_{t}\left(e_{i}\right)$ defined in (3.42). Let $\nabla^{T_{x} M}$ be the flat connection on $T_{x} M$. Let $\Delta^{T_{x} M}$ be the flat Laplacian over $T_{x} M$ for the metric $g^{T_{x} M}$. Using (3.40), (3.46), one concludes easily that as $t \rightarrow 0$, the coefficients of $L_{t}(u)$ converge uniformly over compact sets together with their derivatives to the coefficients of the operator $L_{0}(u)$ given by

$$
\begin{aligned}
L_{0}(u)= & -\Delta^{T_{x} M}-\hat{e}^{0} \widehat{\nabla_{e_{i}} V}(0) \nabla_{e_{i}}^{T_{x} M} \\
& +\frac{1}{8} \sum_{0 \leq i, j \leq 2 m} \sum_{1 \leq k, l \leq 2 m}\left\langle R^{E}\left(e_{i}, e_{j}\right) e_{l}, e_{k}\right\rangle e^{i} e^{j} \hat{e}^{k} \hat{e}^{l} .
\end{aligned}
$$

On the other hand, from (3.28), one has

$$
\frac{\partial}{\partial u} \tilde{D}_{V}(u)=\sqrt{-1} \hat{c}(V) c\left(g^{-1} d g\right) .
$$


Let $C_{t}(u)$ be the operator obtained from $\frac{\partial}{\partial u} \tilde{D}_{V}(u)$ by replacing $c\left(e_{i}\right), \hat{c}\left(e_{i}\right)$ by $c_{t}\left(e_{i}\right), \hat{c}_{t}\left(e_{i}\right)$ respectively.

Set

$$
\omega=g^{-1} d g .
$$

Using (3.50), (3.51), we find that

$$
\lim _{t \rightarrow 0} \sqrt{t} C_{t}(u)=\sqrt{-1} \hat{e}^{0} \sum_{0 \leq i \leq 2 m} \omega\left(e_{i}\right) e^{i} .
$$

Recall that $Q_{t}^{u}(x, y)$ is the kernel of $\frac{\partial}{\partial u} \tilde{D}_{V}(u) \exp \left(-t \tilde{D}_{V}(u)^{2}\right)$.

Let $d v_{M}$ be the volume element on $T M$ with respect to the metric $g^{T M}$. By using Proposition 3.13, Equations (3.49), (3.52), and also the trivial relation $\hat{e}^{0,2}=0$, and by proceeding as in Getzler $[\mathbf{G}]$, we see that as $t \rightarrow 0$, $(3.53)$

$$
\sqrt{t} \operatorname{Tr}\left[Q_{t}^{u}(x, x)\right] d v_{M}(x) \longrightarrow \frac{\sqrt{-1}}{2 \sqrt{\pi}} \operatorname{Tr}\left[g^{-1} d g\right] \operatorname{Pf}\left(\frac{R^{E}}{2 \pi}\right), \quad \text { uniformly on } M \text {. }
$$

This completes the proof of Theorem 3.9. By Remark 3.8, Theorem 3.3 is thus also proved.

Remark 3.14. The local index techniques of this section can also be used to prove regularity results for the $\eta$ functions of $\tilde{D}_{V}(u)$ 's.

\section{Invariants associated to nowhere zero vector fields.}

In this Section, we apply the odd index Theorem 3.3 of the last section to get invariants on odd dimensional manifolds.

Let $M$ as before be an odd dimensional oriented compact manifold.

Let $V$ be a nowhere zero vector field on $M$. Let $\gamma$ be the one dimensional oriented vector bundle generated by $V$. Then $E=T M / \gamma$ is an even dimensional vector bundle over $M$, carrying a canonically induced orientation.

Let $e(E)$ be the Euler class of $E$.

Definition 4.1. Let $\alpha_{V}$ be the homomorphism

$$
\alpha_{V}: H^{1}(M, \mathbf{Z}) \longrightarrow \mathbf{Z}
$$

defined by

$$
\alpha_{V}: \omega \longrightarrow\langle\omega e(E),[M]\rangle,
$$

if $\omega$ is not a torsion class. If $\omega$ is a torsion class, we set $\alpha_{V}(\omega)=0$.

By using Theorem 3.3, we can give an analytic formula for the map $\alpha_{V}$ as follows.

Let $\omega \in H^{1}(M, \mathbf{Z})$. We assume $\omega$ is not a torsion class. Let $\tilde{\omega} \in \wedge^{1}\left(T^{*} M\right)$ be any de Rham representative of $\omega$. 
We fix a point $p$ on $M$. For any $x \in M$, let $\gamma_{p x}$ be a pass connecting $p$ and $x$.

Let $\rho_{\tilde{\omega}}(x): M \rightarrow U(1)$ be the function on $M$ defined by

$$
\rho_{\tilde{\omega}}(x)=\exp \left\{2 \pi \sqrt{-1} \int_{p}^{x} \tilde{\omega}\right\},
$$

where the integral is along the pass $\gamma_{p x}$.

Since $[\tilde{\omega}] \in H^{1}(M, \mathbf{Z}), \rho_{\tilde{\omega}}$ is well defined. Also, one finds

$$
\rho_{\tilde{\omega}}^{-1} d \rho_{\tilde{\omega}}=2 \pi \sqrt{-1} \tilde{\omega} \text {. }
$$

Let $g^{T M}$ be a metric on $T M$ so that $\|V\|_{g^{T M}}=1$. Let $T_{V, \rho_{\tilde{\omega}}}$ be the Toeplitz operator defined for $V, \rho_{\tilde{\omega}}$ as in Section 3a). Then from Theorem 3.3 one deduces easily the following result.

Theorem 4.2. The following identity holds,

$$
\operatorname{ind}\left(T_{V, \rho_{\tilde{\omega}}}\right)=\alpha_{V}(\omega) .
$$

Example 4.3. The simplest example is by taking $M=S^{1} \times X$ for some compact connected oriented even dimensional manifold $X$, and take $V$ to be the canonical vector field lifted from that of $S^{1}$ and $\omega$ the canonical generator of $H^{1}\left(S^{1}, \mathbf{Z}\right)$. Then one has clearly $\alpha_{V}(\omega)=\chi(X)$.

As a simple application to problems involving vector fields, we prove the following result.

Theorem 4.4. If the homomorphism $\alpha_{V}$ is not identically zero, then $V$ can not be deformed through nowhere zero vector fields to $-V$.

Proof. Since $\alpha_{V}$ is not a zero map, there exists $\omega \in H^{1}(M, \mathbf{Z})$ such that

$$
\alpha_{V}(\omega)=\operatorname{ind} T_{V, \omega} \neq 0 .
$$

Now if $V$ is homotopic to $-V$, then by the homotopy invariance of the analytic index, one would have

$$
\operatorname{ind}\left(T_{V, \omega}\right)=\operatorname{ind}\left(T_{-V, \omega}\right)=-\operatorname{ind}\left(T_{V, \omega}\right) .
$$

This contradicts with (4.6).

Remark 4.5. In some cases, Theorem 4.4 gives refined obstructions for a vector field $V$ to be able to homotopic to $-V$. For example, if $M=S^{1} \times X$, $\operatorname{dim} M=4 q+1$, and $V$ is the vector field considered in Example 4.3, then Theorem 2.5 only asserts $\chi(X) \equiv 0(\bmod 2)(\mathrm{cf}$. [A , Theorem 1.3$])$, while Theorem 4.4 asserts that $\chi(X)$ vanishes exactly. 
Remark 4.6. Of course, the map $\alpha_{V}$ can be nontrivial only on nonsimply connected manifolds. For simply connected manifolds, one might expect that the mod 2 indices discussed in Section 2 would provide meaningful invariants.

Remark 4.7. In some sense one may regard Theorem 4.2 as an odd dimensional analogue of the Gauss-Bonnet-Chern theorem. But in line of the above remark, one might also take the mod 2 index theorem [AS2] for the skew-adjoint operator $D_{V}$ constructed in Section 2 as another candidate.

\section{References}

[A] M.F. Atiyah, Vector fields on manifolds, Arbeitsgemeinschaft für Forschung des Landes Nordrhein-Westfalen, 200 (1970), 7-24.

[ABP] M.F. Atiyah, R. Bott and V.K. Patodi, On the heat equations and the index theorem, Invent. Math., 19 (1973), 279-330.

[APS1] M.F. Atiyah, V.K. Patodi and I.M. Singer, Spectral asymmetry and Riemannian geometry I, Proc. Camb. Phil. Soc., 77 (1975), 43-69.

[APS2] _ Spectral asymmetry and Riemannian geometry II, Proc. Camb. Phil. Soc., 79 (1976), 71-99.

[AS1] M.F. Atiyah and I.M. Singer, The index of elliptic operators I, Ann. of Math., 87 (1968), 484-530.

[AS2] ㄴ The index of elliptic operators V, Ann. of Math., 93 (1971), 139-149.

[BD] P. Baum and R.G. Douglas, K-homology and index theory, Proc. Symposia Pure and Appl. Math., Vol. 38, Part I, 117-173, AMS Providence R.I., 1982.

[BGV] N. Berline, E. Getzler and M. Vergne, Heat Kernels and Dirac Operators, SpringerVerlag, 1992.

[BF] J.-M. Bismut and D.S. Freed, The analysis of elliptic families II, Commun. Math. Phys., 107 (1986), 103-163.

[BZ] J.-M. Bismut and W. Zhang, An extension of a theorem by Cheeger and Müller, Astérisque, 205, Paris, 1992.

[BW] B. Booss-Bavnbek and K.P. Wojciechowski, Elliptic Boundary Problems for Dirac Operators, Birkhäuser, Boston, 1993.

[C] S.S. Chern, A simple intrinsic proof of the Gauss-Bonnet formula for closed Riemannian manifolds, Ann. of Math., 45 (1944), 747-752.

[GN] R. Geoghegen and A. Nicas, Trace and torsion in the theory of flows, Topology, 33 (1994), 683-719.

[G] E. Getzler, A short proof of the Atiyah-Singer index theorem, Topology, 25 (1986), 111-117.

[LM] H.B. Lawson and M.-L. Michelsohn, Spin Geometry, Princeton Univ. Press, 1989.

[L] A. Lichnerowicz, Spineurs harmoniques, C.R.A.S. Paris Série A, 257 (1963), 7-9. 
[S] N. Steenrod, The Topology of Fibre Bundles, Princeton Univ. Press, 1951.

Received August 25, 1997. The author was partially supported by Chinese National Science Foundation.

Nankai Institute of Mathematics

Tianjin 300071

People's Republic of China

E-mail address: weiping@sun.nankai.edu.cn 